\title{
ABOUT EXISTENCE OF EQUILIBRIA IN ELECTROMAGNETIC CASTING
}

\author{
BY \\ ANTOINE HENROT AND MICHEL PIERRE \\ Université de Nancy 1, Vandœuvre les Nancy, France
}

1. Introduction. This paper deals with existence of equilibria in a problem arising in electromagnetic casting. A vertically falling column of molten liquid is shaped by a horizontal magnetic field imposed by vertical conductors. We assume that the frequency of the field is so high that it does not penetrate into the liquid and the stirring tendency of the liquid due to the curl of the magnetic force is negligible.

We therefore consider a two-dimensional model as introduced in $[2,8]$ which deals with the mean square values of the various quantities.

We denote by $\Omega_{i}$ the (closed) domain occupied by the liquid and by $\Omega$ its exterior. The current density vector is denoted by $\vec{j}_{0}$ and the total magnetic field by $\vec{B}$. We then have

$$
\begin{aligned}
& \nabla \wedge \vec{B}=\mu_{0} \vec{j}_{0} \text { in } \Omega, \\
& \nabla \cdot \vec{B}=0 \text { in } \Omega \\
& \vec{B} \cdot \vec{n}=0 \text { on } \Gamma=\partial \Omega, \\
& \|\vec{B}\|^{2} / 2 \mu_{0}+\sigma C=\text { constant on } \Gamma, \\
& \vec{B} \text { tends to } 0 \text { at infinity, }
\end{aligned}
$$

where $\mu_{0}$ is the permeability of vacuum, $\vec{n}$ the unit normal vector to the boundary $\Gamma,\|\|$ the euclidian norm, $C$ the curvature of $\Gamma$, and $\sigma$ the superficial tension constant of the liquid. The constant in (1.1.d) is unknown.

In [8], we studied the "inverse" problem corresponding to (1.1), namely: the boundary $\Gamma$ (i.e., the shape of the section) is given, the problem is to find $\vec{j}_{0}$ such that the system (1.1) has a solution $\vec{B}$. Here, we are interested in the direct problem which is a free boundary problem: $\vec{j}_{0}$ is given and we are to find $(\Omega, \vec{B})$ such that (1.1) holds.

This direct problem has been treated explicitly in some particular situations [12] or numerically (see $[1,2,3]$ and also [6], where asymptotic expansions with respect to high frequencies are made in a similar context). Here, we have a different approach

Received June 18, 1990.

(C)1991 Brown University 
which was suggested by the study we made of the inverse problem in [8] and which can be explained as follows.

Assume that (1.1) has a solution where $\Gamma$ is a closed Jordan curve. Then this curve is the image of the unit circle $\Gamma_{0}$ under a mapping $\Phi$ which extends to a holomorphic, univalent mapping of the exterior $\Omega_{0}$ of the unit disk onto $\Omega$. Then, it is easy to see that (see, for instance, [8])

$$
v(x, y)=\ln \left|\Phi^{\prime}(x+i y)\right|
$$

is a solution of the system

$$
\begin{aligned}
& \Delta v=0 \text { on } \Omega_{0}, \\
& -\tau \frac{\partial v}{\partial r}=g^{2} e^{-v}-P e^{v}+\tau \text { on } \Gamma_{0}, \\
& v \text { is bounded at infinity, }
\end{aligned}
$$

where $\tau=2 \sigma \mu_{0}, P$ is the constant appearing in (1.1.d), $\frac{\partial v}{\partial r}$ is the radial derivative, and

$$
g=g\left(e^{i \theta}\right)=\varepsilon \sqrt{P-\tau C}\left|\Phi^{\prime}\left(e^{i \theta}\right)\right|, \quad \varepsilon= \pm 1 .
$$

Note that (1.3.b) is nothing but the boundary condition (1.1.d).

Obviously, $g$ depends on the solution $\Phi$ and is a priori unknown when looking for $\Gamma$. On the other hand, if $g$ were known, we could determine $v$ as the solution of (1.3) and then obtain $\Phi$ (and therefore the free boundary $\Gamma$ ) by integrating (1.2).

We prove in fact, in Sec. 2, that $g$ can be expressed only in terms of $J_{0}=j_{0} \circ \Phi$, the image of $j_{0}$ under $\Phi$. This dependence on $\Phi$ is particularly slight in the case of linear vertical conductors, that is, when $j_{0}$ is given by

$$
j_{0}=\sum_{k=1}^{N} \beta_{k} \delta_{Z_{k}}, \quad \beta_{k} \in \mathbf{R}, Z_{k} \in \mathbf{C},
$$

and $\delta_{Z_{k}}=$ Dirac mass at $Z_{k}$. We then prove that $g$ is given by

$$
g\left(e^{i \theta}\right)=\sum_{k=1}^{N} \alpha_{k}\left\{e^{i \theta} /\left(z_{k}-e^{i \theta}\right)+e^{i \theta} /\left(\bar{z}_{k}-e^{-i \theta}\right)+1\right\},
$$

where

$$
\alpha_{k}=-\beta_{k} \mu_{0} / 2 \pi, \quad \Phi\left(z_{k}\right)=Z_{k}, \quad k=1, \ldots, N .
$$

Therefore, $g$ is completely determined from the knowledge of the $z_{k}$ 's. Here again, this knowledge is implicit since it depends on $\Phi$. However, if, for instance, the $\left(Z_{k}, \beta_{k}\right)$ present some good symmetry property, we expect the $\left(z_{k}, \alpha_{k}\right)$ to have the same. As an example, if the $Z_{k}$ are at the vertices of a square $(N=4)$ and $\beta_{1}=\beta_{3}=-\beta_{2}=-\beta_{4}$, the $z_{k}$ will also be at the vertices of a square. Therefore, the idea to solve the direct problem in this particular case (and it is the scheme of our general approach) is the following:

(i) We take $z_{k}, k=1, \ldots, 4$, at the vertices of a square of side length $a$ and $\alpha_{1}=\alpha_{3}=-\alpha_{2}=-\alpha_{4}=K$.

(ii) We solve (1.3) with $g$ given as in (1.6). 
(iii) We deduce $\Phi$ from integrating (1.2).

(iv) Letting $a$ and $P$ vary, we look at the $\left(Z_{k}, \beta_{k}\right)$ obtained by (1.7) to decide whether a given set can be reached in this way.

Section 2 is devoted to proving that, if a solution exists, then $g$ is completely determined by $J_{0}=j_{0} \circ \Phi$. We write $g=T\left(J_{0}\right)$.

We next assume that $g$ is a given continuous function on $\Gamma_{0}$ and we prove in Sec. 3 that problem (1.3) is well posed, whence the existence of $v$. Then, in Sec. 4, existence of $\Phi$ satisfying (1.2) on $\bar{\Omega}_{0}$ and holomorphic, univalent on $\Omega_{0}$ is analysed. A necessary and sufficient condition for this to hold is produced. If $g=T\left(J_{0}\right)$, and if $J_{0}$ satisfies some good symmetry property, this condition is shown to hold.

The last step would consist of describing the set of functions $\left\{J_{0} \circ \Phi^{-1}\right\}$, where $J_{0}$ varies among all the distribution functions on $\Omega_{0}$ and $\Phi=\Phi\left(J_{0}\right)$ is determined as above. This would provide the initial distributions $j_{0}=J_{0} \circ \Phi^{-1}$ for which an equilibrium exists. This step is rather ambitious in its full generality. However, it can be approached numerically. This will be shown in a coming work, see [5]. As a starting point, we give here (in Sec. 5), some explicit results in the particular situation $\tau=0$, i.e., when the superficial tension is negligible, and when $j_{0}$ is equal to a sum of isolated masses. The curves have been obtained through the same analysis as here. But, if some extra difficulties appear, calculations can be explicitly made. All the details can be found in [9]. Note that in many cases, the case $\tau=0$ is very significant of the more regular situation, $\tau$ positive and small.

We are very grateful to Jean-Pierre Brancher for suggesting this problem. We also thank him for the numerous and fruitful conversations he had with us, especially about the physical aspects of the problem.

2. Determination of $g$. We assume $j_{0}$ to be a real measure with compact support in $\mathbf{R}^{2}$ (identified with the complex plane $\mathbf{C}$ ). The examples we have in mind are of the following form, where $\varphi$ is a continuous test-function on $\mathbf{C}$ :

vertical slender conductors:

$$
\left\langle j_{0}, \varphi\right\rangle=\sum_{k=1}^{N} \beta_{k} \varphi\left(Z_{k}\right), \quad Z_{k} \in \mathbf{C}, \beta_{k} \in \mathbf{R},
$$

measure concentrated on a curve $\gamma$ :

$$
\left\langle j_{0}, \varphi\right\rangle=\int_{\gamma} \varphi(x) j_{0}(x) d \sigma(x),
$$

superficial measure:

$$
\left\langle j_{0}, \varphi\right\rangle=\iint_{K} \varphi(x, y) j_{0}(x, y) \mathrm{dx} \mathrm{dy}, \quad \text { where } K \subset \mathbf{R}^{2} \text { is compact. (2.1.c) }
$$

We will use, for each of these cases, the usual notation:

$$
\left\langle j_{0}, \varphi\right\rangle:=\int_{K} \varphi(x) d j_{0}(x),
$$

where $K$ is the support of the measure $j_{0}$ and is assumed to be compact. 
If $\Phi$ is a holomorphic univalent mapping of the exterior of the unit disk $\Omega_{0}$ onto $\Omega$, we define, as usual, the image $J_{0}$ of $j_{0}$ in the $\Omega_{0}$-plane by

$$
\forall \varphi \in C\left(\Omega_{0}\right) \quad\left\langle J_{0}, \varphi\right\rangle:=\left\langle j_{0}, \varphi \circ \Phi^{-1}\right\rangle,
$$

where $C\left(\Omega_{0}\right)$ denotes the set of real continuous functions on $\Omega_{0}$. Note that the support of the measure $J_{0}$ is $K_{0}=\Phi^{-1}(K)$, which is compact.

We assume now that the problem $(1.1)$ has a solution $(\Omega, \vec{B})$ in the following sense:

There exists a closed Jordan curve $\Gamma$ of class $C^{2}$ and $\Phi \in C^{2}\left(\bar{\Omega}_{0}\right)$, holomorphic on $\Omega_{0}$, and one-to-one on $\bar{\Omega}_{0}$, such that $\Omega=\Phi\left(\Omega_{0}\right)$, $\Omega$ is unbounded, and $\Gamma=\partial \Omega=\Phi\left(\Gamma_{0}\right)$,

$$
\begin{gathered}
K=\text { support of } j_{0} \subset \Omega, \\
\vec{B}=\left(\psi_{y},-\psi_{x}\right) \quad \text { with } \psi \in W_{\mathrm{loc}}^{1,1}(\Omega) \cap C^{1}(\bar{\Omega} \backslash K), \\
-\Delta \psi=\mu_{0} j_{0} \quad \text { in } \mathscr{D}^{\prime}(\Omega), \\
\vec{B} \cdot \vec{n}=0 \quad \text { on } \Gamma(\vec{n} \text { unit normal vector on } \Gamma), \\
\|\nabla \psi\|^{2}+\tau C=P \quad \text { on } \Gamma, \tau=2 \sigma \mu_{0}, P \in \mathbf{R}, C \text { curvature of } \Gamma, \\
\nabla \psi \rightarrow 0 \quad \text { at infinity. }
\end{gathered}
$$

THEOREM 2.1. Let $j_{0}$ be given by (2.2) and assume (2.4)-(2.10) hold. Then $v=$ $\ln \left|\Phi^{\prime}\right|$ is a solution of (1.3), where $g$ is given by

$$
g\left(e^{i \theta}\right)=\alpha \int_{K_{0}}\left\{\frac{e^{i \theta}}{x-e^{i \theta}}+\frac{\bar{x}}{\bar{x}-e^{-i \theta}}\right\} d J_{0}(x), \quad \alpha=-\mu_{0} / 2 \pi,
$$

and where $J_{0}$ is defined by (2.3).

Proof. By (2.4), $\Phi$ has a Laurent expansion of the form

$$
\Phi(z)=C_{1} z+C_{0}+\sum_{n<0} C_{n} z^{n}, \quad C_{1} \neq 0,
$$

with

$$
\Phi^{\prime}(z) \neq 0 \quad \text { on } \Omega_{0}, \quad \lim _{|z| \rightarrow+\infty} \Phi^{\prime}(z)=C_{1} \neq 0 .
$$

Therefore, $v=\ln \left|\Phi^{\prime}\right|$ is the real part of a holomorphic function on $\Omega_{0}$ and satisfies (1.3.a) and (1.3.c).

The relation (1.3.b) can also be easily deduced from (2.9) by using the expression of the curvature of a curve defined as the image of

$$
\theta \rightarrow \Phi\left(e^{i \theta}\right) \quad(\text { see }[8])
$$

and by defining

$$
g\left(e^{i \theta}\right):=\varepsilon \sqrt{P-\tau C}\left|\Phi^{\prime}\left(e^{i \theta}\right)\right|, \quad \varepsilon= \pm 1 .
$$

The precise value of $\varepsilon$ does not matter yet. It is a consequence of the results in [8] that it can be chosen in such a way that $\theta \mapsto g\left(e^{i \theta}\right)$ be analytic. It will also be a consequence of computations below. 
Now the main point of the proof is to establish that the function $g$ is also given by $(2.11)$.

First, (2.6), (2.8) imply that $\psi$ is constant on $\Gamma$. Without loss of generality, we can assume $\psi=O$ on $\Gamma$.

Let $\hat{\psi}$ be defined on $\bar{\Omega}_{0}$ by $\hat{\psi}(z)=\psi(\Phi(z))$ so that $\hat{\psi}$ is a solution of

$$
\begin{cases}-\Delta \hat{\psi}=\mu_{0} J_{0} & \text { on } \Omega_{0}, \\ \hat{\psi}=0 & \text { on } \Gamma_{0} \\ |\nabla \hat{\psi}| \text { tends to } 0 \text { at infinity } & \end{cases}
$$

and also

$$
|\nabla \hat{\psi}|^{2}=(P-\tau C)\left|\Phi^{\prime}\left(e^{i \theta}\right)\right|^{2}=g^{2}\left(e^{i \theta}\right) \quad \text { on } \Gamma_{0} .
$$

The classical integral representation formula of exterior Dirichlet problem (2.13) provides (cf. for instance [7, tome 1, ch. II, §3])

$$
\hat{\psi}(z)=\alpha \int_{K_{0}} \ln \left|\frac{z-\xi}{1-z \bar{\xi}}\right| d J_{0}(\xi),
$$

which is the real part of the function

$$
f(z)=\alpha \int_{K_{0}} \log \left(\frac{z-\xi}{1-z \bar{\xi}}\right) d J_{0}(\xi),
$$

defined and holomorphic in a neighbourhood of $\Gamma_{0}$. We deduce that, on this neighbourhood

$$
f^{\prime}(z)=\hat{\psi}_{x}-i \hat{\psi}_{y}=\alpha \int_{K_{0}}\left(\frac{1}{z-\xi}+\frac{\bar{\xi}}{1-z \bar{\xi}}\right) d J_{0}(\xi) .
$$

From (2.6), (2.8), $\nabla \hat{\psi}$ is normal on $\Gamma_{0}$ and $\hat{\psi} \in C^{1}\left(\bar{\Omega}_{0} \backslash K_{0}\right)$ so that

$$
\hat{\varepsilon}|\nabla \hat{\psi}|=-\nabla \hat{\psi} \cdot \vec{n}=-\left(\begin{array}{c}
\hat{\psi}_{x} \\
\hat{\psi}_{y}
\end{array}\right) \cdot\left(\begin{array}{l}
x \\
y
\end{array}\right)=-z f^{\prime}(z) \quad \text { on } \Gamma_{0} \text {, with } \hat{\varepsilon}= \pm 1 .
$$

Finally (2.17), (2.18), and (2.14) yield

$$
g\left(e^{i \theta}\right)=\varepsilon_{1} \alpha \int_{K_{0}}\left\{\frac{e^{i \theta}}{\xi-e^{i \theta}}+\frac{\bar{\xi}}{\bar{\xi}-e^{-i \theta}}\right\} d J_{0}(\xi),
$$

where $\varepsilon_{1}= \pm 1$. Choosing $\varepsilon_{1} \equiv 1$ determines the choice of $\varepsilon$ in definition (2.12). We then check that $\theta \mapsto g\left(e^{i \theta}\right)$ is analytic.

REMARK 1. Formula (2.11) can also be written as

$$
g\left(e^{i \theta}\right)=\alpha\left\{\mathfrak{R} e\left(\int_{K_{0}} \frac{e^{i \theta}}{\xi-e^{i \theta}} d J_{0}(\xi)\right)+\int_{K_{0}} d J_{0}(\xi)\right\} .
$$

REMARK 2. If $J_{0}=\sum_{k=1}^{N} \alpha_{k} \delta_{z_{k}}, z_{k} \in \Omega_{0}, \alpha_{k} \in \mathbf{R}$, then formula (2.11) becomes

$$
g\left(e^{i \theta}\right)=\alpha \sum_{k=1}^{N} \alpha_{k}\left\{\frac{e^{i \theta}}{z_{k}-e^{i \theta}}+\frac{\bar{z}_{k}}{\bar{z}_{k}-e^{-i \theta}}\right\} .
$$

REMARK 3. For later reference, note that (2.17) gives the derivative of the complex potential $f$ on the component of $\Omega_{0} \backslash K_{0}$ close to $\Gamma_{0}$. 
3. Solving (1.3). We denote by $\mathscr{C}_{b}\left(\bar{\Omega}_{0}\right)$ (resp. $\mathscr{C}\left(\Gamma_{0}\right)$ ) the space of bounded continuous functions on $\bar{\Omega}_{0}$ (resp. $\Gamma_{0}$ ) equipped with the uniform norm

$$
\|u\|_{\infty}=\max _{x \in \bar{\Omega}_{0}}|u(x)| \quad\left(\operatorname{resp} .\|u\|_{\infty, \Gamma_{0}}=\max _{x \in \Gamma_{0}}|u(x)|\right) .
$$

We denote by $\mathscr{C}_{n}^{1}\left(\bar{\Omega}_{0}\right)$ the space of functions $v \in \mathscr{C}_{b}\left(\bar{\Omega}_{0}\right)$ such that the normal derivative $\frac{\partial v}{\partial n}$ is defined and continuous on $\Gamma_{0}$ (for instance in the sense of [7, tome 1 , ch. II, §1]).

Let $g \in \mathscr{C}\left(\Gamma_{0}\right), P>0$ and $\tau>0$. We consider the problem

$$
\left\{\begin{array}{l}
v \in \mathscr{C}_{n}^{1}\left(\bar{\Omega}_{0}\right), \\
\Delta v=0 \quad \text { on } \Omega_{0}, \\
-\tau \frac{\partial v}{\partial n}=P e^{v}-g^{2} e^{-v}-\tau \text { on } \Gamma_{0},
\end{array}\right.
$$

where $\frac{\partial v}{\partial n}=-\frac{\partial v}{\partial r}$ is the normal derivative of $v$ on $\Gamma_{0}$ toward the origin (i.e., exterior to $\Omega_{0}$ ).

We have the following result.

TheOREM 3.1. For $P>0, \tau>0$, and $g \in \mathscr{C}\left(\Gamma_{0}\right), g \not \equiv 0$, the problem (3.1) has a unique solution $v$. The mapping $(P, \tau, g) \mapsto v$ is continuous from $(0, \infty) \times$ $(0, \infty) \times \mathscr{C}\left(\Gamma_{0}\right)$ into $\mathscr{C}_{b}\left(\bar{\Omega}_{0}\right)$, increasing with respect to $g^{2}$ and decreasing with respect to $P$, and the mapping $g \mapsto v$ is compact from $\mathscr{C}\left(\Gamma_{0}\right)$ into $\mathscr{C}_{b}\left(\bar{\Omega}_{0}\right)$ for each $(P, \tau) \in(0, \infty) \times(0, \infty)$. Moreover, if $g^{2}$ is analytic, then $v$ is analytic on $\bar{\Omega}_{0}$ (up to the boundary).

The proof relies on the use of the exterior capacity operator defined on $\mathscr{C}\left(\Gamma_{0}\right)$ and on the perturbation theory for $m$-accretive operators on $\mathscr{C}\left(\Gamma_{0}\right)$. Let us first recall the definition of the exterior capacity operator (see [7, tome 1 , ch. II, §5]) which is a linear unbounded operator on $\mathscr{E}\left(\Gamma_{0}\right)$.

If $\varphi \in \mathscr{C}\left(\Gamma_{0}\right)$, we denote by $u_{e}(\varphi)$ the unique solution of the exterior Dirichlet problem

$$
\begin{cases}u \in \mathscr{C}_{b}\left(\bar{\Omega}_{0}\right), & \\ \Delta u=0 & \text { on } \Omega_{0}, \\ u=\varphi & \text { on } \Gamma_{0} .\end{cases}
$$

We then define the exterior capacity operator $C_{e}$ by

$$
\begin{gathered}
D\left(C_{e}\right)=\left\{\varphi \in \mathscr{C}\left(\Gamma_{0}\right) ; u_{e}(\varphi) \in \mathscr{C}_{n}^{1}\left(\bar{\Omega}_{0}\right)\right\}, \\
C_{e} \varphi=\partial u_{e}(\varphi) / \partial n .
\end{gathered}
$$

With this definition, problem (3.1) is equivalent to

$$
v=u_{e}(\varphi),
$$

where $\varphi$ is a solution of

$$
\tau C_{e} \varphi+P e^{\varphi}-g^{2} e^{-\varphi}=\tau .
$$


Since (3.2) has a unique solution for $\varphi \in \mathscr{C}\left(\Gamma_{0}\right)$ (see [7]), problem (3.1) is equivalent to Eq. (3.6). This one is directly related to the surjectivity of the perturbed nonlinear operator

$$
\varphi \mapsto \tau C_{e} \varphi+\gamma(\cdot, \varphi),
$$

where $\gamma(\cdot, \varphi)=P e^{\varphi}-g^{2}(\cdot) e^{-\varphi}$ is continuous and increasing with respect to $\varphi$. This surjectivity property will be a direct consequence of the properties of the linear operator $C_{e}$ and well-known perturbation results.

The next lemma describes the properties of $C_{e}$ that are needed here. A proof due to $\mathrm{Ph}$. Bénilan can be found in [7, tome 1, ch. II].

We denote by $I$ the identity operator.

Lemma 3.1. Let $\lambda \in \mathscr{C}\left(\Gamma_{0}\right), \lambda \geq 0, \lambda \not \equiv 0$. Then

(i) The operator $\lambda I+C_{e}$ is one-to-one from $D\left(C_{e}\right)$ into $\mathscr{C}\left(\Gamma_{0}\right)$.

(ii) The inverse operator $\left(\lambda I+C_{e}\right)^{-1}$ is continuous and compact from $\mathscr{C}\left(\Gamma_{0}\right)$ into itself.

(iii) $\left(\psi \in \mathscr{C}\left(\Gamma_{0}\right), \psi \geq 0\right) \Rightarrow\left(\lambda I+C_{e}\right)^{-1} \psi \geq 0$.

(iv) $\left(\varphi \in \mathscr{C}\left(\Gamma_{0}\right), x_{0} \in \Gamma_{0}, \varphi\left(x_{0}\right)=\max _{x \in \Gamma_{0}} \varphi(x)\right) \Rightarrow C_{e} \varphi\left(x_{0}\right) \geq 0$.

Here we remark that

$$
\frac{\partial \gamma}{\partial \varphi}(\cdot, \varphi)=P e^{\varphi}+g^{2}(\cdot) e^{-\varphi} \geq 2|g(\cdot)| \sqrt{P}
$$

so that, if we set

$$
\lambda(x):=2 \sqrt{P}|g(x)|, \quad \beta(x, \varphi):=\gamma(x, \varphi)-\lambda(x) \varphi,
$$

then

$$
\varphi \mapsto \beta(x, \varphi) \text { is nondecreasing }
$$

and (3.6) is equivalent to

$$
\lambda \varphi+\tau C_{e} \varphi+\beta(\cdot, \varphi)=\tau .
$$

Lemma 3.2. Let $f \in \mathscr{C}\left(\Gamma_{0}\right)$. Then the problem

$$
\lambda \varphi+\tau C_{e} \varphi+\beta(\cdot, \varphi)=f
$$

has a unique solution. Moreover if $\hat{\varphi}$ is the solution with right-hand side $\hat{f}$, we have

$$
-\left(\lambda I+\tau C_{e}\right)^{-1}\left\{(f-\hat{f})^{-}\right\} \leq \varphi-\hat{\varphi} \leq\left(\lambda I+\tau C_{e}\right)^{-1}\left\{(f-\hat{f})^{+}\right\} .
$$

REMARK. As usual, we denote $r^{+}=\max (r, 0)$ and $r^{-}=\max (-r, 0)$. Relation (3.12) together with Lemma 3.1 proves that

$$
\begin{gathered}
f \mapsto \varphi \text { is increasing, } \\
\|\varphi-\hat{\varphi}\|_{\infty} \leq M\|f-\hat{f}\|_{\infty},
\end{gathered}
$$

where $M$ is the norm of the linear continuous operator $\left(\lambda I+\tau C_{e}\right)^{-1}$ (we have $M \leq\left\|\left(\lambda I+\tau C_{e}\right)^{-1} 1\right\|_{\infty}$, see [7]). 
Proof of Lemma 3.2. We first prove that estimate (3.12) is a consequence of (iv) in Lemma 3.1 and the monotonicity of $\beta$. Indeed, let $\varphi$ and $\hat{\varphi}$ be solutions of (3.11) with data $f, \hat{f}$, let $\psi=\left(\lambda I+\tau C_{e}\right)^{-1}\left\{(f-\hat{f})^{+}\right\}$, and let $x_{0} \in \Gamma_{0}$ such that

$$
(\varphi-\hat{\varphi})\left(x_{0}\right)-\psi\left(x_{0}\right)=\max _{x}\{(\varphi-\hat{\varphi})(x)-\psi(x)\} \text {. }
$$

We have

$$
\left(\lambda I+\tau C_{e}\right)\{(\varphi-\hat{\varphi})-\psi\}+\beta(\cdot, \varphi)-\beta(\cdot, \hat{\varphi})=f-\hat{f}-(f-\hat{f})^{+} \leq 0 .
$$

Therefore, by (iv) of Lemma 3.1 we have

$$
\lambda\left(x_{0}\right)\left\{(\varphi-\hat{\varphi})\left(x_{0}\right)-\psi\left(x_{0}\right)\right\}+\beta\left(x_{0}, \varphi\left(x_{0}\right)\right)-\beta\left(x_{0}, \hat{\varphi}\left(x_{0}\right)\right) \leq 0 .
$$

Since $\beta\left(x_{0}, r\right)$ is nondecreasing and even increasing if $\lambda\left(x_{0}\right)=0$ and since $\psi\left(x_{0}\right) \geq$ $0,(3.17)$ implies that $(\varphi-\hat{\varphi})\left(x_{0}\right)-\psi\left(x_{0}\right) \leq 0$. We deduce the second inequality in (3.12). The first is obtained similarly.

It follows from (3.12) that solutions of (3.11) are unique. For the existence part, we refer to classical results on perturbation of linear operators satisfying the properties of Lemma 3.1 by continuous monotone operators (see [4] or [10]).

Proof of Theorem 3.1. The existence and uniqueness of solutions of (3.1) follow from Lemma 3.2 and the equivalence of (3.1) and (3.5)-(3.6).

For the monotonicity property, if $P \geq \widehat{P}, g^{2} \leq \hat{g}^{2}$, and $\varphi, \hat{\varphi}$ are the corresponding solutions, we write

$$
\lambda \hat{\varphi}+\tau C_{e} \hat{\varphi}+\beta(\cdot, \hat{\varphi})=\tau+(P-\widehat{P}) e^{\hat{\varphi}}-\left(g^{2}-\hat{g}^{2}\right) e^{-\hat{\varphi}} .
$$

Since the right-hand side is greater than $\tau$, we deduce $\varphi \geq \hat{\varphi}$ from (3.13). Thanks to the monotonicity in problem (3.2), we also have

$$
v=u_{e}(\varphi) \geq u_{e}(\hat{\varphi})=\hat{v} .
$$

Let us now establish the continuity property with respect to $g$ (the proof is similar for $P$ and $\tau)$. Let $\hat{g}=g+\varepsilon, \varepsilon \in \mathscr{C}\left(\Gamma_{0}\right)$, where we assume

$$
\|\varepsilon\|_{\infty} \leq\|g\|_{\infty} / 2
$$

so that at $x_{0}$ such that $\left|g\left(x_{0}\right)\right|=\|g\|_{\infty}$ we have

$$
\left|\hat{g}\left(x_{0}\right)\right| \geq\|g\|_{\infty} / 2 \text {. }
$$

Now, one can choose $\hat{\lambda} \in \mathscr{C}\left(\Gamma_{0}\right)$ depending only on $x_{0}$ (i.e., on $g$ ) such that, for all $\varepsilon$ satisfying (3.18)

$$
0 \leq \hat{\lambda} \leq 2 \sqrt{P}|\hat{g}|, \quad \hat{\lambda} \not \equiv 0,
$$

and therefore $r \mapsto \hat{\gamma}(\cdot, r)-\hat{\lambda} r$ is increasing, where $\hat{\gamma}(\cdot, r)=P e^{r}-\hat{g}^{2}(\cdot) e^{-r}$. Thus, if $\hat{\varphi}$ is the solution corresponding to $\hat{g}$, by (3.14) we have

$$
\|\hat{\varphi}\|_{\infty} \leq \widehat{M}\left\|\tau-f_{0}\right\|_{\infty},
$$

where $f_{0}=\hat{\gamma}(\cdot, 0)=P-\hat{g}^{2}$. Therefore, $\hat{\varphi}$ remains uniformly bounded for variations of $\hat{g}=g+\varepsilon$ satisfying (3.18). Now, we remark that

$$
\lambda \hat{\varphi}+\tau C_{e} \hat{\varphi}+\beta(\cdot, \hat{\varphi})=\tau+\left(\varepsilon^{2}+2 \varepsilon g\right) e^{-\hat{\varphi}}
$$


so that, again thanks to (3.14),

$$
\|\varphi-\hat{\varphi}\|_{\infty} \leq M_{1}\|g\|_{\infty}\|\varepsilon\|_{\infty}, \quad \text { where } M_{1}=5 M\left\|e^{-\hat{\varphi}}\right\|_{\infty} / 2 .
$$

Therefore, $g \mapsto \varphi$ is locally Lipschitz continuous. Since $\varphi \mapsto v=u_{\varepsilon}(\varphi)$ is linear continuous, we obtain that $g \mapsto v$ is locally Lipschitz continuous. Compactness of this mapping is an easy consequence of

$$
\varphi=\left(\lambda I+\tau C_{e}\right)^{-1}(\tau-\beta(\cdot, \varphi))
$$

together with compactness of $\left(\lambda I+\tau C_{e}\right)^{-1}$ and continuity of $g \mapsto \varphi$.

The analyticity of $v$, when $g^{2}$ is analytic, has already been noticed and intensively used in [8]. It is a consequence of regularity results for elliptic equations with analytic nonlinear boundary conditions which can be bound in [11].

4. Construction of the free boundary $\Gamma$. According to Theorem 2.1, if the Jordan curve $\Gamma$ solution of $(2.4)-(2.10)$ exists, the corresponding mapping $\Phi$ is such that

$$
v=\ln \left|\Phi^{\prime}\right| \quad \text { on } \bar{\Omega}_{0},
$$

where $v$ is the solution of (3.1) with $g$ given by (2.11). Since $v$ is harmonic on $\Omega_{0}$ and bounded at infinity, there exists $V$ holomorphic on $\Omega_{0}$ and bounded at infinity such that

$$
v=\Re \mathrm{e} V \quad \text { on } \Omega_{0}(\mathfrak{R e}=\text { real part }) .
$$

Indeed $(x, y) \mapsto v(1 /(x+i y))$ is harmonic on $\left\{(x, y) ; 0<x^{2}+y^{2}<1\right\}$ and bounded at 0 ; therefore, it can be extended into a harmonic function on the whole unit disc. The existence of $V$ follows.

Now, if $\Phi$ exists, we necessarily have for some $k \in \Gamma_{0}$

$$
\Phi^{\prime}(z)=k e^{V(z)} \quad \forall z \in \Omega_{0} .
$$

Lemma 4.1. Let $g \in \mathscr{C}\left(\Gamma_{0}\right)$ and let $v$ be the solution of (3.1). Then, there exists $\phi$ holomorphic on $\Omega_{0}$ satisfying (4.3), (4.2) if and only if

$$
\int_{0}^{2 \pi} v\left(e^{i \theta}\right) e^{i \theta} d \theta=0
$$

This is the case if $g$ is given by $(2.11)$ and $\left(J_{0}, K_{0}\right)$ is $\varepsilon$-invariant by a rotation $R_{q}$ of angle $2 \pi / q, q \in \mathbf{N}-\{0,1\}$, in the following sense:

$$
\left\{\begin{array}{l}
\varepsilon= \pm 1, \\
R_{q}\left(K_{0}\right)=K_{0}, \\
\forall z \in K_{0}, J_{0}\left(R_{q} z\right)=\varepsilon J_{0}(z) .
\end{array}\right.
$$

Moreover, if there are two solutions, one obtains the second solution from the first one by a translation and a rotation.

Proof. The function $e^{V(z)}$ is holomorphic on $\Omega_{0}$. It can be written as the derivative of a holomorphic function on $\Omega_{0}$ if and only if the coefficient of $z^{-1}$ in its Laurent expansion is zero. But it is equal to $e^{V_{0}} V_{-1}$, where

$$
V(z)=\sum_{n \leq 0} V_{n} z^{n}
$$


Since

$$
v\left(r e^{i \theta}\right)=\frac{1}{2} \sum_{n \leq 0} r^{n}\left(V_{n} e^{i n \theta}+\bar{V}_{n} e^{-i n \theta}\right), \quad r>1,
$$

we have

$$
V_{-1}=\frac{1}{4 \pi} \int_{0}^{2 \pi} v\left(e^{i \theta}\right) e^{i \theta} d \theta
$$

whence the condition (4.4).

If (4.5) holds, one easily checks that (see (2.11))

$$
g\left(R_{-q} e^{i \theta}\right)=\varepsilon g\left(e^{i \theta}\right) .
$$

Thanks to the uniqueness of the solutions of (3.1) and the invariance of the Laplacian operator by rotation, if $g$ satisfies (4.6), then the corresponding solution $v$ of (3.1) satisfies

$$
v\left(R_{-q} e^{i \theta}\right)=v\left(e^{i \theta}\right) .
$$

By change of variable $\theta=\varphi-2 \pi / q$, we deduce

$$
\int_{0}^{2 \pi} v\left(e^{i \theta}\right) e^{i \theta} d \theta=\int_{0}^{2 \pi} v\left(R_{-q} e^{i \varphi}\right) R_{-q} e^{i \varphi} d \varphi=e^{-2 i \pi / q} \int_{0}^{2 \pi} v\left(e^{i \theta}\right) e^{i \theta} d \theta
$$

Therefore, (4.4) holds.

The last step in constructing $\Gamma$ is to check that the function $\Phi$ obtained from integrating (4.3) (assuming it is possible) will be one-to-one from $\bar{\Omega}_{0}$ onto $\Phi\left(\bar{\Omega}_{0}\right)$. Since $v$ is bounded on $\bar{\Omega}_{0}, \Phi^{\prime}(z)$ does not vanish on $\Omega_{0}$ and is therefore locally injective. But global injectivity remains to be checked. In the case $\tau=0$, we noticed in [9] that $\Phi$, although locally injective, might not be globally injective due to the possibility that $\Phi^{\prime}$ may vanish on $\Gamma_{0}$; this can also happen here as shown by numerical computations, see [5]. However if $\Phi$ is globally injective, it provides a solution of our problem.

To summarize our analysis, we can state the following:

Theorem 4.1. Let $J_{0}$ be a measure with compact support $K_{0}$ in $\Omega_{0}$ and let $g$ be defined by (2.11). Then

(i) There exists $v$, a solution of (3.1).

(ii) Assume moreover that $J_{0}$ satisfies invariance property (4.5). Then there exists $\Phi$ holomorphic in a neighbourhood of $\bar{\Omega}_{0}$ and unique up to a rotation or a translation such that $v=\ln \left|\Phi^{\prime}(x+i y)\right|$ satisfies (3.1).

(iii) If moreover $\Phi$ is univalent on $\bar{\Omega}_{0}$, it is a solution of problem (2.4)-(2.10) with $j_{0}$ defined by $(2.3)$ and with the potential $\psi$ given by

$$
\psi(Z)=\mathfrak{R e}\left(f\left(\phi^{-1}(Z)\right)\right),
$$


with $\mathfrak{R e} f$ is defined on $\Omega_{0}$ by

$$
\Re \operatorname{e} f(z)=\int_{K_{0}} \ln \left|\frac{z-\xi}{1-z \bar{\xi}}\right| d J_{0}(\xi)
$$

REMARK 1. The analyticity of $\Phi$ on a neighbourhood of $\bar{\Omega}_{0}$ can be deduced from the analyticity of $v=\ln \left|\Phi^{\prime}\right|$ on $\Gamma_{0}$, see [8].

REMARK 2. If we assume the simple extra assumption: $\Gamma=\Phi\left(\Gamma_{0}\right)$ is without double point (i.e., $\Phi$ is univalent on $\Gamma_{0}$ ), then we can prove that $\Phi$ is univalent on $\bar{\Omega}_{0}$, see [5].

5. Examples. The curves we present here have been obtained by the above analysis, in the particular case $\tau=0$ (i.e., superficial tension negligible) which explains the appearance of cusps in Figs. 3 and 5.

This hypothesis makes the problem (3.1) degenerate in an exterior Dirichlet problem on the unit circle whose explicit resolution is easy. All details and formulae can be found in [9].

In the cases of Figs. 1, 2, 4, and 6, the function $g$, defined in (1.6), does not vanish on the unit circle. We can then prove that these curves are obtained as the limit as $\tau$ tends to zero of corresponding curves with $\tau>0$. Therefore they are quite representative of the more regular situation, $\tau$ positive and small, studied in this article. If $g$ vanishes, the behaviour as $\tau$ tends to 0 is not so obvious. At least, cusps can occur as in Figs. 3 and 5.

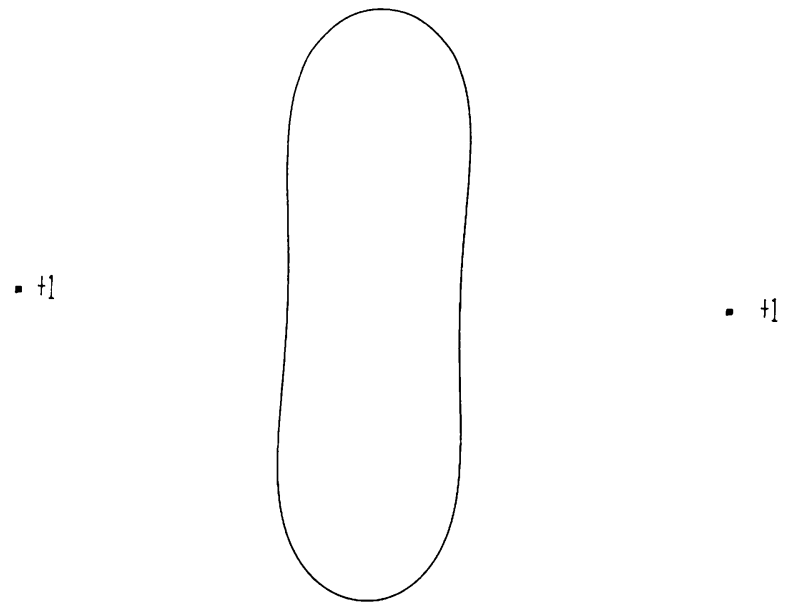

Fig. 1. Two conductors, same sign. 
$-1$

- +1

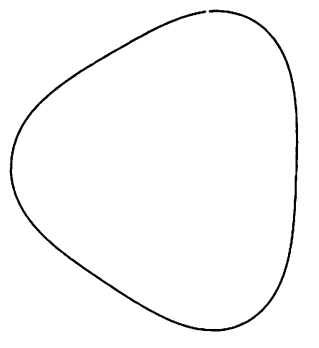

- +1

Fig. 2. Three conductors, same sign.
- +1

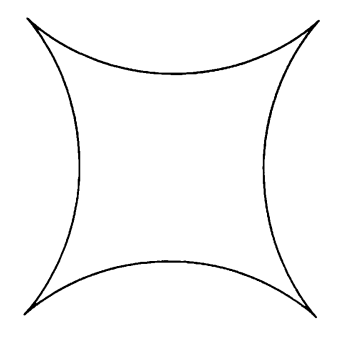

- -1

- +1

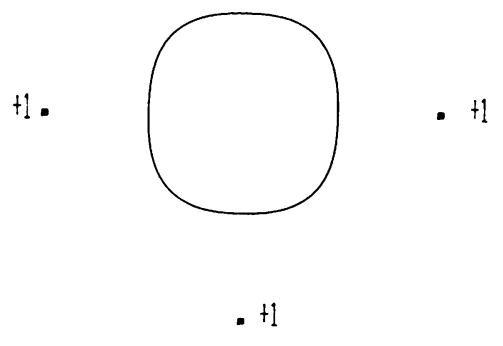

Fig. 4. Four conductors, same sign.

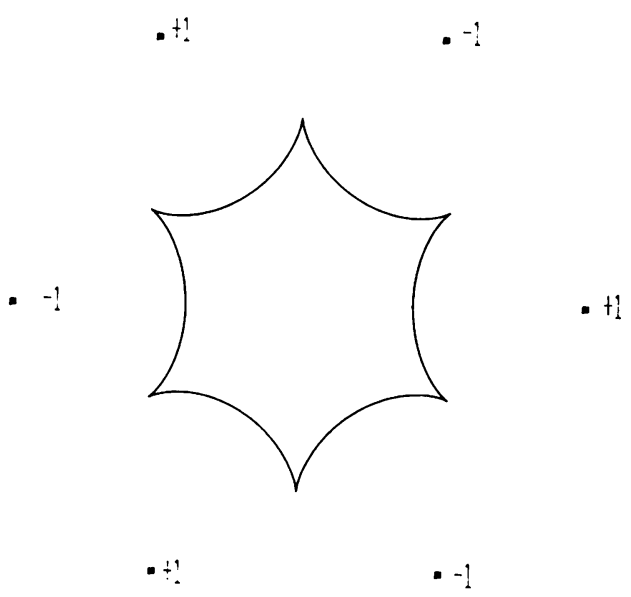

Fig. 5. Six conductors, alternate sign. 
- +1

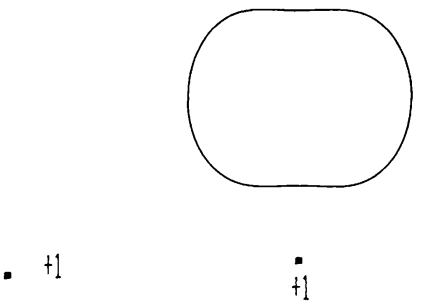

+1 .

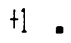

Fig. 6. Six conductors, same sign.

Acknowledgments. This work was supported by the following Institutions: University of Nancy I; URA CNRS 750; INRIA Lorraine, Projet NUMATH.

\section{REFERENCES}

[1] J.-P. Brancher, R. De Framond, and O. Sero-Guillaume, Shaping of liquid metal cylinders, 4th Beer Sheva Seminar on MHD Flows and Turbulence, Israel, 1984

[2] J.-P. Brancher, J. Etay, and O. Sero-Guillaume, Formage d'une lame, J. Méc. Théor. Appl. 2 (6), 976-989 (1983)

[3] J.-P. Brancher and O. Sero-Guillaume, Sur l'équilibre des liquides magnétiques. Application à la magnétostatique, J. Méc. Theor. Appl. 2 (2), 265-283 (1983)

[4] H. Brezis and W. A. Strauss, Semi-linear second order elliptic equations in $L^{1}$, J. Math. Soc. Japan 25, 565-590 (1973)

[5] O. Coulaud and A. Henrot, Numerical study of a free boundary problem arising in electromagnetic casting (to appear)

[6] M. Crouzeix and J. Descloux, A bidimensional electromagnetic problem, SIAM J. of Math. Anal. 21 (3), , 577-592 (1990)

[7] R. Dautray and J.-L. Lions, Analyse mathématique et Calcul numérique pour les sciences et les techniques, Vol. 1, Masson, 1984

[8] A. Henrot and M. Pierre, Un problème inverse en formage des métaux liquides, RAIRO Modél. Math. Anal. Numer. 23 (1), 155-177 (1989)

[9] A. Henrot and M. Pierre, About existence of a free boundary in electromagnetic shaping, Recent Advances in Nonlinear Elliptic and Parabolic Problems, vol. 208, Pitman Research Notes Series Pitman, New York, 1989

[10] Y. Konishi, Semi-linear Poisson's equations, Proc. Japan Acad. 49, 100-105 (1973)

[11] C. B. Morrey., Jr., Multiple Integrals in the Calculus of Variations, Springer-Verlag, 1966

[12] J. A. Shercliff, Magnetic shaping of molten metal columns, Proc. Roy. Soc. London Ser. A. 375, 455-473 (1981) 